\title{
Elastografía cuantitativa en la evaluación de nódulos tiroideos
}

\section{Quantitative Elastography in the Evaluation of Thyroid Nodules}

\author{
Carolina Uliaque Franco ${ }^{1}$ Ricardo Herrero Laborda ${ }^{2}$ Elena Hervias Ángulo ${ }^{1}$ Ana Almenara Riaguas ${ }^{1}$ \\ Francisco Javier Berdún Pardo ${ }^{1}$ \\ ${ }^{1}$ Servicio de Radiodiagnóstico, Centro Médico de Especialidades Ramón y
Cajal, Hospital Universitario Miguel Servet, Zaragoza, España
${ }^{2}$ Centro Universitario de la Defensa, Zaragoza, España \\ Address for correspondence Carolina Uliaque Franco, Médico \\ Especialista en Radiodiagnóstico, C/Miguel de Cervantes, \\ $15,3^{\circ} \mathrm{B}$, Zaragoza 50006, España \\ (e-mail: caroluliaque@hotmail.com).
}

Rev Argent Radiol 2021;85:83-90.

\section{Resumen \\ Palabras clave \\ - Sonoelastografía \\ - Nódulos tiroideos \\ - Biopsia \\ - Aspiración con aguja fina \\ - Regresión logística}

\section{Abstract}

received

October 9, 2019

accepted

October 9, 2020
Objetivo Evaluar retrospectivamente la capacidad diagnóstica de la elastografía cuantitativa para determinar la posibilidad de malignidad o benignidad de los nódulos tiroideos benignos y malignos, y determinar su utilidad para así decidir qué nódulos deberán ser sometidos a punción aspirativa con aguja fina (PAAF).

Pacientes y métodos Se analizaron 203 nódulos tiroideos de 195 pacientes remitidos por el Servicio de Endocrinología para estudio citológico durante el año 2018. A todos ellos se les realizó ecografía convencional, elastografía cuantitativa y PAAF. Se realizó un análisis estadístico mediante regresión logística, que pone en relación la probabilidad de que un nódulo sea sospecho de malignidad y el valor de la elasticidad medido en kilopascales ( $\mathrm{kPa}$ ) y el ratio elastográfico.

Resultados Existe una relación significativa y positiva entre el resultado citológico de Bethesda V/VI y los kPas/ratio elastográfico. Se recomienda realizar PAAF a aquellos nódulos con valores superiores a $25 \mathrm{kPa}$ y/o ratio elastográfico superior a 1,5.

Conclusión La elastografía cuantitativa es una herramienta útil que, junto a otros parámetros ecográficos, ayudaría a predecir o sospechar la malignidad de un nódulo tiroideo y a una mejor selección para la PAAF.

Objective To retrospectively assess the diagnostic capacity of quantitative elastography to determine the odds between benign and malignant thyroid nodules, and determine its usefulness in deciding which nodules should be subjected to fine needle aspiration puncture (FNA).

Patients and methods 203 thyroid nodules from 195 patients referred by the Endocrinology Service for cytological study during the year 2018 were analyzed. All
DOI https://doi.org/

$10.1055 / \mathrm{s}-0040-1721347$. ISSN 1852-9992.

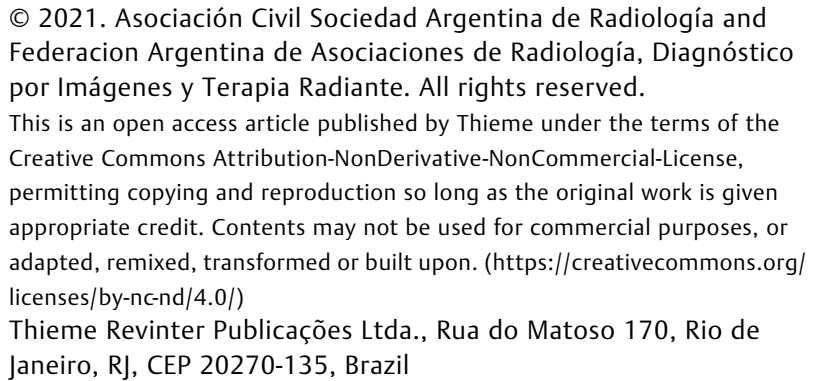

(C) 2021. Asociación Civil Sociedad Argentina de Radiología and Federacion Argentina de Asociaciones de Radiología, Diagnóstico por Imágenes y Terapia Radiante. All rights reserved. This is an open access article published by Thieme under the terms of the Creative Commons Attribution-NonDerivative-NonCommercial-License, permitting copying and reproduction so long as the original work is given appropriate credit. Contents may not be used for commercial purposes, or adapted, remixed, transformed or built upon. (https://creativecommons.org/ licenses/by-nc-nd/4.0/)

Thieme Revinter Publicações Ltda., Rua do Matoso 170, Rio de Janeiro, RJ, CEP 20270-135, Brazil 
Keywords

- Sonoelastography

- Thyroid nodules

- Biopsies

- Fine-needle aspiration

- Logistic regression of them underwent conventional ultrasound, quantitative elastography and FNA. A statistical analysis was performed using logistic regression that relates the probability that a nodule is suspected of malignancy and the elasticity value measured in kilopascals ( $\mathrm{kPa}$ ) and the elastographic ratio.

Results There is a significant and positive relationship between the cytological result of Bethesda V / VI and the kPas / elastographic ratio. FNA is recommended for those nodules with values greater than $25 \mathrm{kPa}$ and / or elastographic ratio greater than 1.5. Conclusion Quantitative elastography is a useful tool that, together with other ultrasound parameters, would help to predict the malignancy of a thyroid nodule and to better select for FNA.

\section{Introducción}

En la actualidad, existe un importante aumento del número de nódulos tiroideos diagnosticados por ecografía, debido a una mayor demanda de pruebas diagnósticas por parte de médicos y pacientes, que hace indispensable la disposición de una herramienta diagnóstica no invasiva, que nos ayude a determinar qué nódulos deben ser biopsiados y cuáles son susceptibles de seguimiento. ${ }^{1-3}$ La preponderancia de la punción aspirativa con aguja fina (PAAF) como prueba de elección en el diagnóstico de nódulos tiroideos, para diferenciar entre benignidad y malignidad, presenta limitaciones. Por un lado, dicha prueba no se puede realizar a todos los nódulos de tiroides diagnosticados, debido a que el número elevado de casos existentes implica un consumo de recursos económicos y de tiempo excesivos, generando asimismo un costo adicional en términos de estrés para el paciente. Por otro lado, la PAAF es una prueba invasiva, que presenta posibles complicaciones (infecciones, hematomas, reacciones vasovagales, hemorragias), está sujeta a errores en la toma de la muestra, y no exenta de presentar falsos negativos hasta en un $15 \%$ en pacientes con cáncer. ${ }^{1,4-8}$

En este artículo, analizamos la elastografía como método complementario en la evaluación de la malignidad de los nódulos tiroideos para determinar la necesidad de realizar la PAAF. Se trata de una prueba de imagen avanzada, no invasiva, que mide la elasticidad o rigidez de los tejidos porque se basa en la evidencia de que los tumores malignos son más rígidos que los nódulos benignos y que el tejido tiroideo normal. ${ }^{9-12}$

Nuestro objetivo es analizar la utilidad de la elastografía cuantitativa, a través del valor de la elasticidad del nódulo medido en $\mathrm{kPa}$ o conforme al ratio elastográfico, como prueba independiente para detectar el cáncer de tiroides, de manera que pueda ser utilizada para disminuir y optimizar el número de procedimientos invasivos realizados.

\section{Pacientes y métodos}

Se trata de un estudio retrospectivo y observacional, respetando los principios de la Declaración de Helsinki 1975, con la revisión de octubre de 2000. Se obtuvieron los consentimientos informados necesarios de los pacientes implicados en el estudio para la realización de todas las pruebas y el consentimiento del Comité de Ética.

\section{Pacientes}

Se incluyeron 203 nódulos tiroideos de 195 pacientes consecutivos, remitidos por el Servicio de Endocrinología para su estudio citológico mediante PAAF ecodirigida durante un periodo de un año, desde enero a diciembre del 2018. Los endocrinos siguieron como recomendación los criterios de inclusión de la Guía de la Asociación de Endocrinología Clínica y de la Asociación Americana del tiroides. ${ }^{13-15}$

Se descartaron del estudio dos nódulos tiroideos por limitaciones técnicas, debido a la imposibilidad para realizar la PAAF, ya que no se pudo acceder a ellos por su localización en la encrucijada cervicotorácica. Los dos pacientes fueron sometidos a cirugía.

Tras la primera PAAF, en 25 ocasiones obtuvimos un resultado no diagnóstico, que nos obligó a realizar una segunda punción, tras la cual seis de ellos obtuvieron un diagnóstico de benignidad, mientras que en los 19 restantes persistió el resultado no diagnóstico.

\section{Ecografía y elastografía}

Los estudios fueron realizados por dos radiólogos con amplia experiencia, utilizando un ecógrafo Toshiba Aplio 500 dotado de elastografía cuantitativa.

A todos los pacientes se les realizó en primer lugar una ecografía convencional, en escala de grises (modo B) y Doppler color, en tiempo real. Se utilizó una sonda lineal de alta frecuencia $(7-12 \mathrm{MHz})$. Posteriormente, se llevó a cabo la elastografía cuantitativa, llamada así porque ofrece información medible y objetiva de la elasticidad de los tejidos. Mide la velocidad de propagación de las ondas tangenciales (shear waves) en los tejidos. Esas ondas se producen en sentido perpendicular a las ondas longitudinales, son mucho más lentas que ellas y su velocidad es proporcional a la elasticidad de los tejidos, de manera que atraviesan los tejidos rígidos a mayor velocidad. La elasticidad se expresa en unidades de velocidad en $\mathrm{m} / \mathrm{sg}$ o como presión en kilopascales $(\mathrm{kPa}) .{ }^{7,11,16}$

La principal diferencia con la elastografía cualitativa es que no funciona a tiempo real ni se requiere ejercer compresión externa, porque mide el desplazamiento 

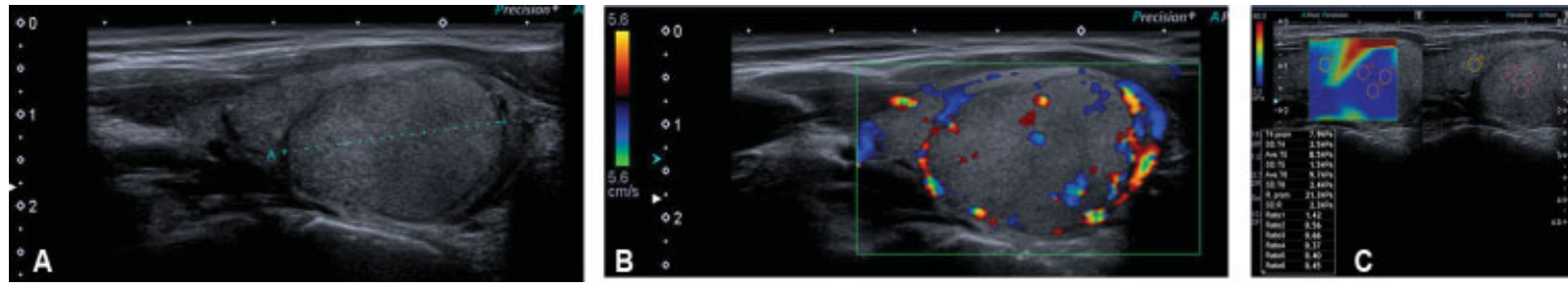

Fig. 1 (A) Mujer de 65 años con lesión nodular sólida en lóbulo tiroideo derecho y diagnóstico citológico de Bethesda II, lesión benigna. Presenta ecoestructura homogénea e hipoecoica, bien delimitada con halo hipoecoico alrededor. (B) El Doppler color arroja vascularización de predominio periférico. (C) La elastografía presenta un mapa de colores predominantemente azul oscuro con valores bajos de Kilopascales, inferiores a 10, y ratios elastográficos menores a 1 , lo que refleja que el nódulo es más blando que el tejido tiroideo normal adyacente. Los hallazgos ecográficos y elastográficos sugieren lesión benigna.

independientemente de la presión aplicada, al enviar microimpulsos acústicos con niveles mínimos de energía hacia los diferentes tejidos. ${ }^{16,17} \mathrm{Su}$ mayor ventaja es que disminuye la variabilidad inter e intraobservador, porque es una prueba no operador dependiente. ${ }^{16,18,19}$

El paciente debe mantenerse quieto, sin hablar, tragar ni respirar, para evitar artefactos de movimiento, y la sonda debe permanecer perpendicular a la piel, inmóvil e intentando evitar su posición oblicua. ${ }^{5-10}$

La técnica de elastografía viene integrada en el ecógrafo. Cuando se selecciona esa aplicación, aparece una caja en la imagen en modo $B$, que se debe colocar en tiempo real sobre el tejido del que se desea obtener un valor cuantitativo de elasticidad. La caja debe incluir el nódulo a estudiar y tejido tiroideo normal. Se obtiene un mapa de colores de los diferentes tejidos incluidos dentro de la caja que, según su grado de elasticidad, son: azul para los tejidos muy blandos, rojo para los muy rígidos, verde y amarillo para los valores intermedios de elasticidad. ${ }^{7-20}$ Se toman varias medidas mediante la colocación de una región de interés (ROI) dentro del nódulo y una medida de referencia del tejido tiroideo normal. Se evitará incluir dentro de la ROI las zonas quísticas y las calcificaciones. $^{5-7}$ Algunos autores recomiendan la realización de 10 mediciones para obtener un valor fiable. ${ }^{21}$ Sin embargo, otros autores han demostrado que no existe diferencia significativa entre la realización de cinco o 10 mediciones para valorar la elasticidad de los tejidos. ${ }^{19}$ No obstante, nosotros efectuamos en todos los casos 10 mediciones. El cociente que resulta de la comparación entre la deformidad tisular en la lesión y el del tejido normal da el valor del ratio elastográfico.

El aumento de rigidez de un tejido se correlaciona con el aumento de velocidad de la onda de ultrasonidos y con el aumento de presión medida en $\mathrm{kPa} .^{19-22}$

En las siguientes figuras presentamos tres casos diferentes, con imágenes de ecografía en modo B, doppler color y elastograma: -Figura 1a, 1b, 1c; -Figura 2a, 2b, 2c; - Figura 3a, 3b, 3c.

En una segunda cita programada, se procedió a la toma de muestras a través de una PAAF. Para ello, se emplearon agujas de calibre 21-22G conectadas a una jeringa de 20cc y un mecanismo de aspiración (Cameco Medical Ltd London NW6 2 BP). Se envió la muestra al Servicio de Anatomía Patológica y el patólogo basó su diagnóstico citológico en la clasificación de Bethesda 2017 (- Tabla 1). ${ }^{23}$

\section{Métodos de análisis estadístico}

Realizamos un análisis de regresión logística que pone en relación la probabilidad de que un nódulo sea sospechoso de malignidad o maligno (resultado citológico Bethesda V/VI) y el valor de la elasticidad del nódulo medido en $\mathrm{kPa} o$ conforme al ratio elastográfico. Asimismo, se calculan los odds ratios y una medida del ajuste total del modelo (pseudo$R^{2}$ ). Hacemos especial hincapié en la capacidad de los modelos estimados para clasificar correctamente los nódulos malignos. Para ello, calculamos la sensibilidad, especificidad, valor predictivo positivo y negativo (VPP y VPN) para cada modelo.
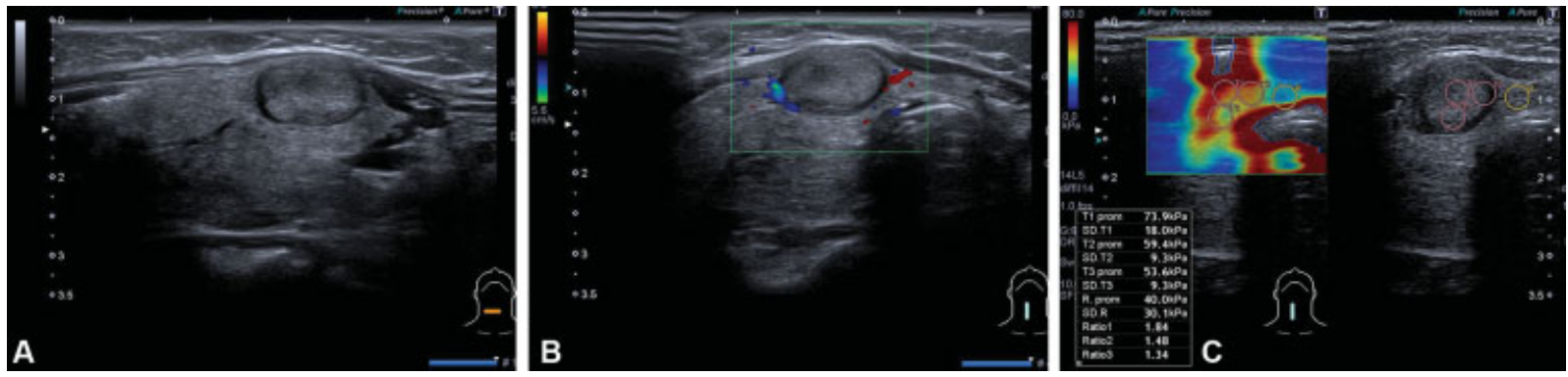

Fig. 2 (A y B) Mujer de 52 años con lesión nodular sólida en lóbulo tiroideo derecho, de aspecto ecográfico benigno: bien delimitada con halo hipoecoico, de ecoestructura homogénea e hipoecoica y con vascularización escasa y de predominio periférico demostrado con el Doppler color. (C) La elastografia, a diferencia de la paciente anterior, muestra un patrón rígido con valores altos de kilopascales, sospechoso de malignidad. Gracias al aporte de la elastografía, se tomó la decisión de realizar PAAF. El resultado anatomopatológico fue de Bethesda V, sospechoso de malignidad. 

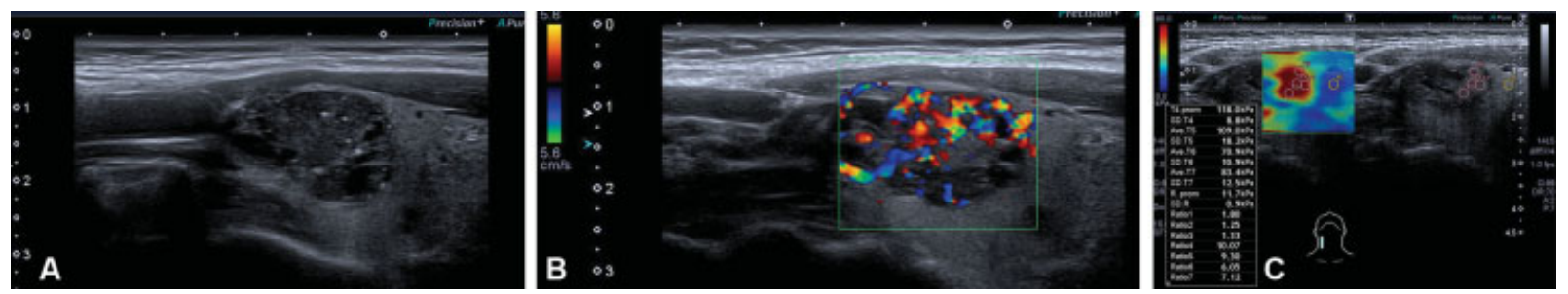

Fig. 3 (A) Mujer de 48 años con lesión nodular en el lóbulo tiroideo derecho, de contornos bien definidos, ecoestructura heterogénea, con pequeñas áreas coloides y microcalcificaciones. (B) Con el Doppler color presenta un patrón de vascularización central aumentada. (C) En la elastografía se colocaron los ROI en la parte sólida del nódulo, representada en el mapa de colores como un área de máxima rigidez, rojo intenso, con valores altos de kipopascales, que oscilan entre 80 y 120, con un ratio de 6 a 10 (media de 8,1). Los hallazgos ecográficos y elastográficos eran sospechosos de malignidad. El diagnóstico citológico fue de Bethesda $\mathrm{V}$, sospechosa de carcinoma papilar.

Tabla 1 Actualización del Sistema Bethesda 2008/2017

\begin{tabular}{|l|l|l|}
\hline & 2008 & 2017 \\
\hline Categoría diagnóstica & Riesgo de cáncer (\%) & Riesgo de cáncer (\%) \\
\hline I) Muestra insuficiente & Repetir PAAF & Repetir PAAF \\
\hline II) Resultado benigno & $0-3$ & $0-3$ \\
\hline III) Lesión folicular o de significado incierto & $5-15$ Repetir PAAF & $10-30$ Repetir PAAF \\
\hline IV) Neoplasia folicular o probable neoplasia folicular & $15-30$ & $25-40$ \\
\hline V) Diagnóstico presuntivo de cáncer & $60-75$ & $50-75$ \\
\hline VI) Resultado maligno & $97-99$ & $97-99$ \\
\hline
\end{tabular}

Tabla 2 Frecuencia relativa de los nódulos de acuerdo a la clasificación Bethesda. Media y deviación típica de Kpa y Ratio elastográfico

\begin{tabular}{|l|l|l|l|l|l|}
\hline & \multicolumn{2}{|l|}{ kPa } & \multicolumn{2}{l|}{ Ratio elastográfico } \\
\hline & Frecuencia relativa & Media & Desviación típica & Media & Desviación típica \\
\hline Bethesda I & $9,36 \%$ & 15,6 & 7,5 & 1,1 & 0,5 \\
\hline Bethesda II & $74,88 \%$ & 18,9 & 10,8 & 1,3 & 0,6 \\
\hline Bethesda III & $4,93 \%$ & 31,5 & 26,9 & 2,3 & 0,9 \\
\hline Bethesda IV & $4,93 \%$ & 23,7 & 22,1 & 1,9 & 1,2 \\
\hline Bethesda V & $4,93 \%$ & 62,6 & 35,2 & 4,0 & 2,4 \\
\hline Bethesda VI & $0,99 \%$ & 75,5 & 6,4 & 5,4 & 0,4 \\
\hline
\end{tabular}

Tabla 3 Resultado del análisis de regresión logística para la detección de nódulos tiroideos malignos a partir del valor de Kpa

\begin{tabular}{|l|l|l|l|l|}
\hline & $\boldsymbol{\beta}$ & $\mathbf{z}$ & Odds ratio & pseudo- $\boldsymbol{R}^{2}$ \\
\hline Bethesda V o VI & 0,07 & 5,02 & 1,07 & 0,41 \\
\hline
\end{tabular}

\section{Resultados}

El estudio incluye 203 nódulos tiroideos, de los cuales 19 obtuvieron un resultado Bethesda I o no diagnóstico en las dos punciones realizadas, 152 presentaron un diagnóstico citológico Bethesda II o benigno, 10 fueron Bethesda III o atipia de significado incierto, 10 neoplasia foliculares o Bethesda IV, 10 sospechosos de malignidad o Bethesda V, y 2 obtuvieron un resultado maligno o Bethesda VI.

En la - Tabla 2 presentamos la frecuencia relativa de los nódulos de acuerdo a la clasificación Bethesda, y observamos que dos terceras partes de los nódulos son benignos y elásticos con valores de presión bajos, por debajo de $20 \mathrm{kPa}$ de media y un ratio elastográfico medio de 1,3. Por otra parte, los nódulos sospechosos de malignidad o malignos suponen aproximadamente el $6 \%$, con valores altos de presión, por encima de $60 \mathrm{kPa}$ (de media entre 62 y $75 \mathrm{kPa}$ ) con un ratio elastográfico medio de 4 a 5,4.

Realizamos un análisis de regresión logística que proporciona un coeficiente $\beta$ que relaciona la probabilidad de que un nódulo sea maligno con el valor observado de $\mathrm{KPa}$. La - Tabla 3 muestra que existe una relación positiva y significativa entre ambas variables, de manera que un mayor valor de presión tiene asociado una mayor probabilidad de malignidad del nódulo bajo estudio. El modelo tiene una capacidad elevada de explicación porque el pseudo- $R^{2}$ es elevado (41\%), sobre todo teniendo en cuenta que se ha estimado un modelo univariante. 


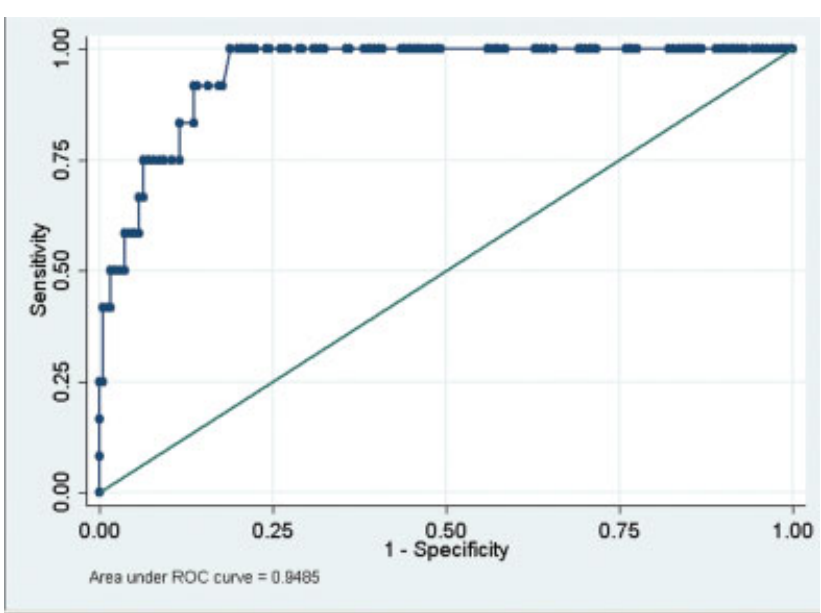

Fig. 4 Curva ROC utilizando el valor Kpa como variable explicativa de la probabilidad de malignidad del nódulo tiroideo.

Tabla 4 Resultado del análisis de regresión logística para la detección de nódulos tiroideos malignos a partir del valor de ratio elastográfico

\begin{tabular}{|l|l|l|l|l|}
\hline & $\boldsymbol{\beta}$ & $\mathbf{z}$ & Odds ratio & pseudo- $\boldsymbol{R}^{2}$ \\
\hline Bethesda V o VI & 1,48 & 4,87 & 4,40 & 0,44 \\
\hline
\end{tabular}

Para los diferentes puntos de corte o probabilidad que permite clasificar un nódulo como sospechoso de malignidad, calculamos la sensibilidad y especificidad del modelo que nos permite calcular el área debajo de la curva ROC (Receiver Operating Characteristic) (-Figura 4), que se interpreta como la probabilidad de clasificar correctamente a un paciente elegido al azar. El valor es muy elevado, aproximadamente igual a 0,95. Valores reducidos de la probabilidad de corte deparan de forma conjunta una sensibilidad y especificidad elevadas.

De acuerdo con el modelo estimado, se recomienda realizar PAAF a todos aquellos nódulos con valores igual o superior a $25 \mathrm{kPa}$. La probabilidad de corte asociada a dicho valor (3\%) clasifica correctamente un $82 \%$ de los nódulos con una sensibilidad del $100 \%$ y una especificidad del $80 \%$. El VPP es del $24 \%$ y el VPN del $100 \%$. De esa forma, se obtiene una garantía elevada de realizar PAAF a los nódulos que efectivamente son malignos y se reducen considerablemente el número de nódulos no malignos a los que se le realiza dicha prueba (38 de 191).

Realizamos el mismo análisis de regresión logística utilizando el ratio elastográfico como variable explicativa de la probabilidad de malignidad de un nódulo tiroideo, obteniendo resultados muy similares a los obtenidos con la variable Kpa. La - Tabla 4 muestra que existe una relación positiva y significativa entre la probabilidad de malignidad del nódulo bajo estudio y el valor del ratio elastográfico (valor del pseudo- $R^{2}$ igual a 0,46 ). Asimismo el área debajo de la curva ROC (- Figura 5) es de nuevo aproximadamente igual a 0,95.

Según el modelo estimado, se recomienda realizar PAAF cuando el ratio elastográfico es superior a 1,5. La probabilidad

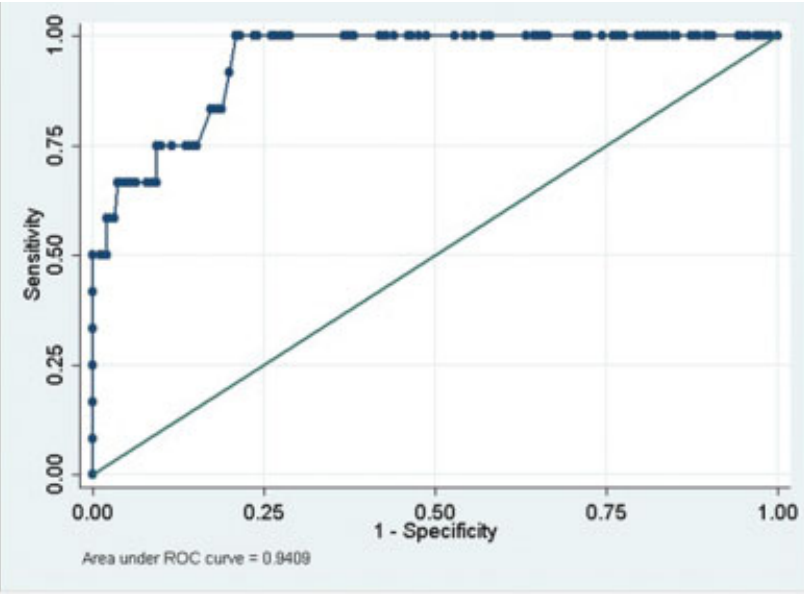

Fig. 5 Curva ROC utilizando el valor del ratio elastográfico como variable explicativa de la probabilidad de malignidad del nódulo tiroideo.

de corte asociada a dicho valor (3\%) clasifica correctamente un $73 \%$ de los nódulos con una sensibilidad del 100\% y una especificidad del $71 \%$. El VPP es del $18 \%$ y el VNN del $100 \%$ De esa forma, se obtiene una garantía elevada de realizar PAAF a los nódulos que efectivamente son malignos y se reducen considerablemente el número de nódulos no malignos a los que se realiza dicha prueba (54 de 191).

\section{Discusión}

Se estima que hasta un $68 \%$ de la población presenta nódulos tiroideos, siendo en su mayoría benignos, con una baja prevalencia de cáncer de tiroides, de aproximadamente el $5 \%{ }^{5-20}$ La ecografía es la prueba inicial utilizada en el diagnóstico de nódulos tiroideos, pero presenta limitaciones porque se ha demostrado que no existen hallazgos ecográficos patognomónicos. ${ }^{24}$ Los criterios ecográficos sospechosos de malignidad varían considerablemente de un artículo a otro, existiendo distintos protocolos de actuación ante el diagnostico de un nódulo tiroideo en los diferentes centros hospitalarios. La PAAF es la prueba de elección que determina qué nódulos deben ser intervenidos quirúrgicamente (aquellos con diagnóstico citológico Bethesda III, IV, V y VI). ${ }^{5,6}$ Se necesitan pruebas diagnósticas que nos ayuden a elegir qué nódulos tiroideos analizados por ecografía deben ser sometidos a PAAF para reducir los costos asociados a dicha prueba.

Nuestro estudio, con base en métodos estadísticos, ha demostrado que la elastografía cuantitativa puede cumplir adecuadamente ese objetivo. En ese sentido, existen estudios que avalan nuestras conclusiones. Samir y col., ${ }^{25}$ abogan por la utilidad de elastografía cuantitativa en el diagnóstico preoperatorio de malignidad de las lesiones foliculares. Mehrotra y col., ${ }^{26}$ concluyen en su estudio que aquellos nódulos tiroideos que son blandos en la elastografía tienen una alta probabilidad de no ser neoplásicos, y por tanto la elastografía llevada a cabo por parte de un operador experimentado puede ser un medio útil para evitar la biopsia de los nódulos benignos. De esa forma, los nódulos que resulten 
intermedios o duros en la elastografía se deberían someter a biopsia.

También hay autores que discrepan con nuestros resultados. Ippolito y col., 7 realizan un estudio sobre 214 pacientes que no logra confirmar el papel potencial de la elastografía en la diferenciación entre nódulos tiroideos benignos y malignos.

Los resultados de nuestro trabajo contrastan con parte de la literatura, que sugiere que la elastografía es útil únicamente en el diagnóstico de nódulos benignos. Kagoya y col., ${ }^{1}$ afirman que la elastografía solo juega un papel importante en el diagnóstico de lesiones tiroideas benignas, y siempre en conjunto con los hallazgos ecográficos en modo B. Luo y col., 27 muestran en su artículo que su método de triaje basado en la elastografía tiene el potencial de reducir sustancialmente el número de biospias mediante la detección de los nódulos benignos. Nell y col., ${ }^{28}$ afirman que la mayor fortaleza de la elastografía es la detección de benignidad, especialmente cuando el nódulo es completamente blando, lo que puede ser calificado seguramente como benigno.

Es interesante recalcar que previamente habíamos realizado, en el mismo centro, dos estudios sobre la elastografía de carácter cualitativo, donde no encontramos una asociación estadísticamente significativa entre los patrones de elastografía y la malignidad/benignidad de los nódulos tiroideos. ${ }^{29,30}$ Hecho que también describen otros autores en sus artículos, y que se debe fundamentalmente a que la elastografía cualitativa es una técnica subjetiva y con baja reproductibilidad, obteniendo resultados elastográficos diferentes sobre un mismo nódulo según el grado de fuerza ejercido. ${ }^{10,18,31,32}$ Esa limitación de la elastografia cualitiativa, no está presente en la elastografia cuantitativa.

En nuestro estudio, sugerimos como valor de corte para recomendar PAAF, $25 \mathrm{kPa}$ con una sensibilidad del 100\%, especificidad del $80 \%$, VPP del $24 \%$ y VPN del $100 \%$. En la literatura encontramos distintos estudios con otros valores razonablemente similares. Samir y col., ${ }^{25}$ en su estudio realizado con un equipo de ultrasonidos GE Logiq E9, establecen el valor de corte en $22,3 \mathrm{kPa}$, con una sensibilidad de $82 \%$, especificidad de $88 \%$, VPP de $75 \%$ y VPN de $91 \%$. Liao y col., ${ }^{33}$ utilizando el mismo equipamiento que en nuestro estudio (Toshiba Aplio 500), establecen como valor de corte $32 \mathrm{kPa}$, con una sensibilidad del $81 \%$, una especificidad del $65 \%$, un VPP del 23\% y un VPN del 96\%, de forma que confirma la elastografía como un predictor independiente de malignidad de los nódulos tiroideos.

Otros autores prefieren utilizar el ratio elastográfico como parámetro para establecer el valor de corte. Nuestra recomendación es realizar la PAAF cuando el ratio elastográfico sea igual o mayor de 1,5 , porque en nuestra muestra dicho valor depara una sensibilidad del 100\%, especificidad del $71 \%$, VPP del $18 \%$ y VPN del $100 \%$. Cantisani y col., ${ }^{34}$ establecen un valor de corte superior del ratio elastográfico, 2,02, con una sensibilidad del 93\% y especificidad del $92 \%$. Kura y col., ${ }^{16}$ fijan como punto de corte elastográfico que permite descartar patología tiroidea maligna menor o igual a 2, con un VPN del 99\% y un VPP del $15 \%$. El incremento del ratio elastográfico por encima de ese valor se asocia a una mayor probabilidad de patología maligna, pero no puede establecer un valor de corte debido al bajo número de casos Bethesda V-VI en su serie. En definitiva, nuestra recomendación de realizar la PAAF cuando el ratio elastográfico sea igual o mayor de 1,5 es más conservadora que en artículos previos. Sin embargo, como se puede ver en la - Tabla 3 evitaríamos realizar la PAAF en media a los Bethesda de tipo I y II, que obviamente constituyen el mayor porcentaje de la muestra, de forma que lograríamos cumplir nuestro objetivo de reducir las PAAF cuando sean claramente innecesarias.

De acuerdo a los resultados de nuestro trabajo, sugerimos que sería interesante usar los datos proporcionados por la elastografía cuantitativa, mediante el valor de la elasticidad del nódulo medido en kPa o conforme al ratio elastográfico, junto al resto de parámetros ecográficos sospechosos de malignidad que se han demostrado estadísticamente significativos en nuestro artículo previo: la presencia de microcalcificaciones, la vascularización de predominio central, una marcada hipoecogenicidad y la morfología del nódulo con un diámetro anteroposterior (alto) mayor que trasversal (ancho). ${ }^{35}$ Existen otros artículos que también afirman que la elastografía debería combinarse con los hallazgos ecográficos obtenidos en modo B, para incrementar la sensibilidad y especficidad de la prueba diagnóstica. 3,6,36

Nuestro estudio tiene como principal limitación que los resultados se basan en diagnósticos citológicos mediante PAAF y no en estudios histológicos, que son el gold estándar para confirmar la benignidad o malignidad de los nódulos tiroideos. Otra limitación es la baja prevalencia de cáncer de tiroides (5\%), que podría afectar la robustez de nuestras conclusiones.

\section{Conclusión}

La elastografía cuantitativa, a través del valor de la elasticidad del nódulo medido en kPa o conforme al ratio elastográfico, ayuda a predecir la malignidad de los nódulos tiroideos. De esa forma, la elastografía se erige como una herramienta radiológica que debería ocupar un lugar definitivo e importante dentro del algoritmo diagnóstico del nódulo tiroideo. Asimismo, hemos mostrado cómo la elastografía cuantitativa permite optimizar la selección de pacientes que deberían ser sometidos a una PAAF.

\section{Responsabilidades Éticas}

Protección de personas y animales. Los autores declaran que para esta investigación no se han realizado experimentos en seres humanos ni en animales.

Confidencialidad de los datos. Los autores declaran que han seguido los protocolos de su centro de trabajo sobre la publicación de datos de pacientes.

Derecho a la privacidad y consentimiento informado. Los autores declaran que en este artículo no aparecen datos de pacientes. 


\section{Nota}

Los autores garantizan que la investigación respeta las pautas éticas de la institución a la que pertenecemos, y la Declaración de Helsinki de 1975, con la revisión de octubre de 2000.

\section{Conflictos de Intereses}

Los autores declaran no tener ningún conflicto de intereses.

\section{Referencias}

1 Kagoya $\mathrm{R}$, Monobe $\mathrm{H}$, Tojima $\mathrm{H}$. Utility of elastography for differential diagnosis of benign and malignant thyroid nodules. Otolaryngol Head Neck Surg. 2010;143(02):230-234

2 Manso S, Velasco MJ. Valor actual de la ecografía en la caracterizacion de los nódulos tiroideos. Revisión de las últimas guías clínicas de actuación. Radiologia (Madr). 2015;57(03):248-258

3 Ríos A, Rodríguez JM, Torregrosa NM, Torregrosa B, Cepero A, Abellán MD, et al. Evaluación del nódulo tiroideo con ecografía y elastografía de alta resolución sin la punción-aspiración con aguja fina. Med Clin (Barc). 2018;151(03):89-96

4 Gennisson JL, Deffieux T, Fink M, Tanter M. Ultrasound elastography: principles and techniques. Diagn Interv Imaging. 2013;94(05):487-495

5 Liu BJ, Lu F, Xu HX, Guo LH, Li DD, Bo XW, et al. The diagnosis value of acoustic radiation force impulse (ARFI) elastography for thyroid malignancy without highly suspicious features on conventional ultrasound. Int J Clin Exp Med. 2015;8(09):15362-15372

6 Paredes-Manjarrez C, Magdalena-Buitrago A, Meza-Hernández G, Camacho-Zarco E, Avelar-Garnica FJ, Tanus-Hajj J, GuerreroRivera S. Elastografía en la evaluación de los nódulos tiroideos. Rev Med Inst Mex Seguro Soc. 2017;55(Suppl 4):S402-S407

7 Ippolito D, Galimberti S, Leni D, Vacirca F, Nasr A, Bragazzi NL, et al. Use of Shear Wave Elastography in the Sonographic Triage of Thyroid Nodules: Feasibility Study in a Series of Lesions Already Selected for Fine Needle Aspiration. J Ultrasound Med. 2019;38 (07):1713-1720

8 Hambleton C, Kandil E. Appropriate and accurate diagnosis of thyroid nodules: a review of thyroid fine-needle aspiration. Int J Clin Exp Med. 2013;6(06):413-422

9 Rago T, Santini F, Scutari M, Pinchera A, Vitti P. Elastography: new developments in ultrasound for predicting malignancy in thyroid nodules. J Clin Endocrinol Metab. 2007;92(08):2917-2922

10 Moon HJ, Sung JM, Kim EK, Yoon JH, Youk JH, Kwak JY. Diagnostic performance of gray-scale US and elastography in solid thyroid nodules. Radiology. 2012;262(03):1002-1013

11 Guzman F, Abellán D, Reus M. La elastografia: una nueva aplicación de la ecografia. ¿Cuál es su utilidad clínica? Radiologia (Madr). 2014;56(04):290-294

12 McQueen AS, Bhatia KS. Thyroid nodule ultrasound: technical advances and future horizons. Insights Imaging. 2015;6(02):173-188

13 Gharib H, Papini E, Valcavi R, Baskin HJ, Crescenzi A, Dottorini ME, et al.AACE/AME Task Force on Thyroid Nodules. American Association of Clinical Endocrinologists and Associazione Medici Endocrinologi medical guidelines for clinical practice for the diagnosis and management of thyroid nodules. Endocr Pract. 2006;12(01):63-102

14 David S Cooper, Gerard M Doherty, Bryan R Haugen, Richard T Kloos, Stephanie L Lee. et al.American Thyroid Association (ATA) Guidelines Taskforce on Thyroid Nodules and Differentiated Thyroid Cancer. Revised American Thyroid Association management guidelines for patients with thyroid nodules and differentiated thyroid cancer. Thyroid. 2009;19(11):1167-1214

15 Haugen BR, Alexander EK, Bible KC, Doherty GM, Mandel SJ, Nikiforov YE. 2015 American Thyroid Association Management Guidelines for Adult Patients with Thyroid Nodules and
Differentiated Thyroid Cancer: The American Thyroid Association Guidelines Task Force on Thyroid Nodules and Differentiated Thyroid Cancer. Thyroid. 2016;26(01):1-133

16 Kura M, Ballarino C, Tamagnone F, Campagno B, Bertini K, Gómez Escalante J, Vega A, Peressotti B, Vilallonga J, Saubidet A. Relación entre el valor del ratio elastográfico y la clasificación citológica de Bethesda en la patología tiroidea. Rev Argent Radiol. 2014;78(03): 128-137

17 Melodelima D, Bamber JC, Duck FA, Shipley JA. Transient elastography using impulsive ultrasound radiation force: a preliminary comparison with surface palpation elastography. Ultrasound Med Biol. 2007;33(06):959-969

18 Park SH, Kim SJ, Kim EK, Kim MJ, Son EJ, Kwak JY. Interobserver agreement in assessing the sonographic and elastographic features of malignant thyroid nodules. AJR Am J Roentgenol. 2009;193(05):W416-W423

19 Fukuhara T, Matsuda E, Donishi R, Koyama S, Miyake N, Fujiwara K, et al. Clinical efficacy of novel elastography using acoustic radiation force impulse (ARFI) for diagnosis of malignant thyroid nodules. Laryngoscope Investig Otolaryngol. 2018;3(04):319-325

$20 \mathrm{Hu}$ X, Liu Y, Qian L. Diagnostic potential of real-time elastography (RTE) and shear wave elastography (SWE) to differentiate benign and malignant thyroid nodules: A systematic review and metaanalysis. Medicine (Baltimore). 2017;96(43):e8282

21 Bojunga J, Dauth N, Berner C, Meyer G, Holzer K, Voelkl L, et al. Acoustic radiation force impulse imaging for differentiation of thyroid nodules. PLoS One. 2012;7(08):e42735

22 Gu J, Du L, Bai M, Chen H, Jia X, Zhao J, Zhang X. Preliminary study on the diagnostic value of acoustic radiation force impulse technology for differentiating between benign and malignant thyroid nodules. J Ultrasound Med. 2012;31(05):763-771

23 Cibas ES, Ali SZ. The 2017 Bethesda System for reporting thyroid cytopathology. Thyroid. 2017;27(11):1341-1346

24 Nachiappan AC, Metwalli ZA, Hailey BS, Patel RA, Ostrowski ML, Wynne DM. The thyroid: review of imaging features and biopsy techniques with radiologic-pathologic correlation. Radiographics. 2014;34(02):276-293

25 Samir AE, Dhyani M, Anvari A, Prescott J, Halperm EF, Faquin WC, et al. Shear-Wave elastography for the preoperative risk stratification of follicular-patterned lesions of the thyroid: diagnostic accuracy and optimal measurement plane. Radiology. 2015;277(02):565-573

26 Mehrotra P, McQueen A, Kolla S, Johnson SJ, Richardson DL. Does elastography reduce the need for thyroid FNAs? Clin Endocrinol (Oxf). 2013;78(06):942-949

27 Luo S, Kim EH, Dighe M, Kim Y. Thyroid nodule classification using ultrasound elastography via linear discriminant analysis. Ultrasonics. 2011;51(04):425-431

28 Nell S, Kist JW, Debray TP, de Keizer B, van Oostenbrugge TJ, Borel Rinkes $\mathrm{IH}$, et al. Qualitative elastography can replace thyroid nodule fine-needle aspiration in patients with soft thyroid nodules. A systematic review and meta-analysis. Eur J Radiol. 2015;84(04):652-661

29 Franco C, Pardo J, Laborda R, Pérez C. Utilidad de la elastografía para predecir la malignidad de los nódulos tiroideos. Radiologia (Madr). 2016;58(05):366-372

30 Franco C, Pardo FJ, Laborda R, Pérez C. Aplicación de la técnica de árboles de clasificación y regresión en la valoración ecográfica de los nódulos tiroideos. Rev Argent Radiol. 2017; 81(01):17-27

31 Lippolis PV, Tognini S, Materazzi G, Polini A, Mancini R, Ambrosini $\mathrm{CE}$, et al. Is elastography actually useful in the presurgical selection of thyroid nodules with indeterminate cytology? J Clin Endocrinol Metab. 2011;96(11):E1826-E1830

32 Rivo Vaázquez A Rodríguez Lorenzo A Rivo Vázquez JE, Páramo Fernández García-Lorenzo F Pardellas Rivera H, et al. The use of ultrasound elastography in the assessment of malignancy risk in thyroid nodules and multinodular goitres. Clin Endocrinol (Oxf). 2013;79(06):887-891 
33 Liao LJ, Chen HW, Hsu WL, Chen YS. Comparison of Strain Elastography Shear Wave Elastography, and Conventional Ultrasound in Diagnosing Thyroid Nodules. J Med Ultrasound. 2019;27(01):26-32

34 Cantisani V, Grazhdani H, Ricci P, Mortele K, Di Segni M, D’Andrea V, et al. Q-elastosonography of solid thyroid nodules: assessment of diagnostic efficacy and interobserver variability in a large patient cohort. Eur Radiol. 2014;24(01):143-150
35 Franco C, Pardo J, Laborda R, Pérez C. Utilidad de la ecografía en la evaluación de los nódulos tiroideos. Radiologia (Madr). 2016;58 (05):380-38

$36 \mathrm{Xu} \mathrm{JM}, \mathrm{Xu} \mathrm{XH}, \mathrm{Xu} \mathrm{HX}$, Zhang YF, Zhang J, Guo LH, et al. Conventional US, US elasticity imaging, and acoustic radiation force impulse imaging for prediction of malignancy in thyroid nodules. Radiology. 2014;272(02):577-586 